\title{
Refining of used Engine Oil
}

\author{
Sreehari Nair ${ }^{\mathrm{a}}$, Uddhav Pai ${ }^{\mathrm{b}}$, Senroid Fernandes ${ }^{\mathrm{c}}$, Satyam Prabhudesai ${ }^{\mathrm{d}}$, Joshua Sapeco ${ }^{\mathrm{e}}$, \\ Sharad Shanbhag ${ }^{\mathrm{f}}$, Swapnil Ramani ${ }^{\mathrm{g}}$, Sanjeel Naik ${ }^{\mathrm{h}}$ \\ a, b,c,d,e Don Bosco College Of Engineering, \\ Goa, Student. Mechanical Eng. Dept, India \\ f,g,h Don Bosco College Of Engineering, \\ Goa, Faculty. Mechanical Eng. Dept, India
}

\begin{abstract}
This paper presents the result of Recycling of used engine oil using glacial acetic acid, kaolinite and activated charcoal. After a certain period of useful life, the lubricating oil loses its properties and cannot be used as such in machinery. Build up of temperature degrade the lubricating oil ,thus leading to reduction in properties such as viscosity, density, flash point etc. Dirt and metal parts worn out from the surfaces are also deposited into the lubricating oils. with increased time of usuage, the lubricating oil loses its lubricating properties as a result over reduction of desired properties, it must be replaced with a fresh one.The lubricating oil consist of $98 \%$ Base oil and $2 \%$ additives,the base oil never gets spoiled,it only gets dirty hence using the refining process the contaminants and the additives are separated from the base oil. It is a device that receives used engine oil and refines devalued properties of the oil close to its original valued properties. The process includes various steps like supplementation of glacial acetic acid, mixing, centrifuging, mixing of kaolinite dehydration and bleaching using activated charcoal.The unit is semi-automated,it is programmed by using a arduino board,Testing of the used engine oil and the refined engine oil was carried out sucessfully, the refining of used engine oil with acetic acid-clay treatment was carried out sucessfully resulting in red transparent colour base oil.This process of refining of used engine oil did not emit poisonous gases like sulphur dioxide to the atmosphere.In addition the glacial acetic acid has less impact on the processing equipment compared with sulphuric acid.This method produces base oil comparable to that produced using conventional methods.
\end{abstract}

\section{Keywords - Engine oil, base oil, Kaolinite, Charcoal}

\section{INTRODUCTION}

Waste engine oil is a high pollutant material that requires responsible management. Waste engine oil may cause damage to the environment when dumped into the ground or into water streams including sewers. This may result in ground water and soil contamination. Recycling of such contaminated materials will be beneficial in reducing engine oil costs thus causing significant positive impact on the environment. The conven- tional methods of recycling of waste engine oil either requires a high cost technology such as vacuum distillation or the use of toxic materials such as sulfuric acid. These methods also produce contaminating by-products which have high sulphur levels, re-refining of used lube oil is an econom- ically attractive recycling method in terms of resources conservation and environment protection.it allows processing of hazardous material in a safe and effective way to recover a high quality oil product. This results in a strong economic incentive for rerefining considering oil price. Re-refining can produce base oil group 1 or group 2 that is suitable feed stock to refin- ery units. Used lube oil is generally a mixture of different types and grades of used lube oils, coming from motor crankcases and industry users. Used lube oil is made up of multitude of small individual batches collected at garages maintenance shops, transportation companies and industries and depends on local situation seasonal consumption collection source and or- Refining of Used Engine Oil Lubricant oils have been used primarily for reducing friction between moving parts of various machinery or equipment, minimize mate- rial wear, improve the efficiency of equipment machinery and for fuel and energy savings. Access to lubricants is essential to any modern society and not only does lubrication reduce friction and wear by interposition of a thin liquid film between moving surfaces, but it also removes heat, keeps equipment clean, and prevents corrosion. Waste lubricating oil refers to the engine oil, transmission oil, hydraulic and cutting oils after use. These oils must be changed and removed from the automobile after a few thou- sand kilometers of driving because of stress from serious deterioration in service. The amount of lubricating oils that is collected annually in India is very large, approximately 1.7 to 3.5 million tons. This large amount of waste engine oils has a significant impact on both economic and environmental aspects. If discharged into the land, water or even burnt as a low grade fuel, this may cause serious pollution problems because they release harmful metals and other pollutants into the environment.

\section{LITERATURE REVIEW}

\subsection{Refining of used Engine oil using M.E.K}

Mr. Merai Yash P. worked on refining of used engine oil and having carried out the following process:

Process: 1 - Dehydration The oil is heated to $130 \rightarrow C$ in a closed vessel to boil off emulsified water and some of the fuel diluents. The point at which oil contains the maximum amount of dissolved water is termed the saturation point Higher the temperature, higher is the saturation point and hence more water held in solution, in the dissolved phase. Similarly, older the oil, higher is the level of water that can be dissolved. Water is a generator of other contaminants in the oil such as waxes, suspensions, carbon and oxide insoluble and even micro-organisms, so it is removed by dehydration.

Process: 2 - Vacuum Distillation The vacuum distillation column internals must provide good vaporliquid contacting while, at the same time, maintaining a very low pressure increases from the top of the column top to the bottom. Therefore, the vacuum column uses distillation trays only where withdrawing products from the side of the column (referred to as side draws). Most of the column uses packing material for the vapor- liquid contacting because such packing has a lower pressure drop than

distillation trays. This packing material can be either structured sheet metal or randomly dumped packing's. The dehydrated oil is then fed continuously into a vacuum 
distillation plant for fractional in exactly the same fashion as crude petroleum. The fractions obtained are as follows:

1. Light fuel and diesel: It can produce enough diesels from the used oil feed stock to run all the burners and boilers, giving total self-sufficiency in fuel.

2. Lubricating oil: The bulk of the feed stock will distill off in the plant to produce a lubricating oil fraction.

3. Residue: The non-distillable part of the feed stock. This contains all the carbon, wear metals, degraded additives and most of the lead and oxidation products. This residue is successfully used as bitumen extender for roading.

Process: 3 -- Solvent extraction and final atmospheric distillation Methyl Ethyl Ketone (MEK) is a selective aromatic solvent employed in the solvent extraction process. The lubricating oil obtained by vacuum dis- tillation is mixed by agitation with MEK in ratio of 2:1. The lubricating oil and solvent mixture is allowed to settle in separator tank. The aromatic content and degraded additives present in the lubricating oil fraction will settle at the bottom and the lubricating oil fraction and solvent mixture layer forms at the top. Solvent mixture is again subjected to atmospheric distillation. The atmospheric distillation is carried out at temperature of around $80 \rightarrow \mathrm{C}$ which is the boiling point of MEK. The MEK vapor produced is condensed and is again used as solvent by blending with fresh solvent The lubricating oil produced at this stage is similar to that of the base lubricating oil. Additives have to be added to further improve the prop-erties and to make them eligible for use in the automobile engines. Zinc Dialkyl Dithiosulphate is the common additive added to the lubricating oil.

Conclusion: Re-refining of waste lubricants could result in both envi- ronmental and economic benefits. Re-refining of waste oil to manufacture base oil conserves more energy than reprocessing the waste oil for use as fuel. The energy required to manufacture re-refined oil from used oil is only one-third of the energy required to refine crude oil to produce virgin base oil. Therefore, re-refining is considered by many as a preferred option in terms of conserving resource as well as minimizing waste and reducing damage to the environment.

\subsection{Refining of used Engine oil using Bleaching Agents}

Mr. Abdulkadir presents the result of regeneration of used engine oil using industrial bleaching earth and activated carbon as the bleaching agents. The used oil undergoes acidification and bleaching steps to remove the aromatic content and to improve the color and quality of the oil. The pro- cess showed a better quality of regenerated oil with a viscosity of $53.16 \mathrm{cP}$ at 40 Celsius The processes are as follows: Acid Treatment: $300 \mathrm{ml}$ of used engine oil was measured in a $500 \mathrm{ml}$ beaker. Also, $30 \mathrm{ml}$ of sulphuric acid was measured in a separate $50 \mathrm{ml}$ beaker. The regulator hot plate was switched on and the measured base oil was placed on top. The temperature of the base oil (used engine oil) was maintained at $40-45 \rightarrow$ C. At this temperature the sulphuric acid was introduced into the used engine oil simultaneously with stirring of the mixture for 10 minutes. Sedimentation /Decantation At the end of the acid treatment step, the acidic oil was allowed to settle 4 hours to form sediment at the bottom of the beaker. After this period, the acidic-oil was properly sedimented and was decanted into another $500 \mathrm{ml}$ beaker while the residue i.e acidic sludge at the bottom of the beaker was discarded.

Bleaching: The acidic oil in the beaker was then subjected to bleach- ing. The oil was placed on a regulator hot plate and the temperature was maintained at a temperature of $110 \rightarrow$ C. $6 \mathrm{wt} \%$ of bleaching earth (industrially produced) was introduced into the oil and the mixture was continuously stirred for 10 minutes. At the end of the bleaching step, the bleached oil was neutralized.

Neutralization: The bleached oil was neutralized to adjust the $\mathrm{pH}$ of the oil to neutrality. At this step, $4 \mathrm{wt} \%$ of the oil of hydrated lime was introduced into the bleached oil by taken into consideration the $\mathrm{pH}$ of the bleached oil at a given point in time. The bleached oil was neutralized with a continuous manual stirring for 10 minutes. At the end of the bleaching and neutralization steps, the oil was allowed to sediment in the beaker for 4 hours and was decanted into the beaker, while the residue at the bottom of beaker was discarded.

Bleaching using activated Carbon: At the end of the acid treat- ment step, if the acidic oil is to be bleached using activated carbon (char- coal), the same procedure for bleaching using industrial bleaching earth is also used. Except that the temperature was increased and maintained at a range of $130 \Leftrightarrow \mathrm{C}-140 \rightarrow \mathrm{C}$. The same amount of activated carbon as that of the industrial bleaching earth and hydrated lime was used. Sedimentation /Decantation:During this stage, the oil was allowed to sediment in the beaker for 4 hours and was decanted into another beaker, while the residue at the bottom of the beaker was discarded.

Filtration: The sedimented oil was finally filtered using a filter cloth and the filtrate was collected in a filtration flask and was observed to be clear while, the residue (filter cake) was discarded.

Conclusion: From the result of experiment obtained, used engine oil can be regenerated on a bench scale at controlled conditions.It showed a better quality of regenerated oil using industrial bleaching earth with a viscosity at $400 \rightarrow C$, specific gravity and total acid number so close to that of the standard grade.

\subsection{Refining of used Engine oil using a New Washing}

Agent Ihsan Hamawand, Talal Yusaf and Sardasht Rafat have worked on refining of used engine oil and having carried out the following process: This paper addresses recycling of waste engine oils treated using acetic acid. The advantage of using the acetic acid is that it does not react with base oils. The recycling process takes place at room temperature. The recycling process started with removing the contamination from the used engine oil. Glacial acetic acid was mixed with the used engine oil using a ratio of 0.8 $\mathrm{mL}$ acetic acid to $10 \mathrm{~mL}$ used engine oil. This was followed by stirring in a closed container at room conditions (room temperature and pressure) for one hour. The mixture was left for 24 hours at room conditions and then underwent centrifugation for one hour to separate the base oil from the contaminants. The separated base oil was then mixed with kaolinite at a ratio of $1 \mathrm{~mL}$ oil to $4 \mathrm{~g}$ kaolinite. The kaolinite was added to remove the dark color and the smell which result from oxidation of some components in the oil. This 
was achieved by heating to a temperature slightly higher than $250 \rightarrow \mathrm{C}$, followed by centrifugation for $30 \mathrm{~min}$. This process produced a yellow clear base oil and an amount of sludge very close to the initial weight of the kaolinite used plus $5 \mathrm{wt} \%$. Conclusion: This research has shown that used engine oil can be recycled by using glacial acetic acid. This method produces base oil com- parable to that produced using conventional methods.

\section{III.WORKING METHODOLOGY}

The Base oil never gets spoiled,it only gets dirty hence using the glacial acetic acid-clay treatment we are separating the base oil from the contam- inants and the additives.

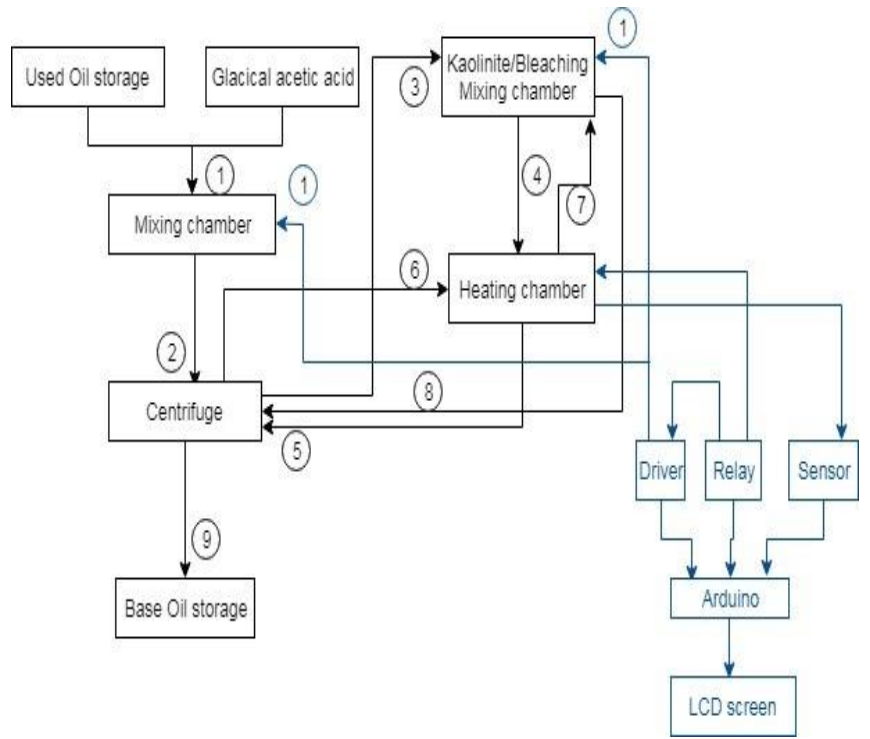

PROCESS PROFILE

1) Supplementation of Glacial acetic acid:Glacial acetic acid is mixed with the used engine oil with proportional ratios(For 2 litres of used engine oil $160 \mathrm{ml}$ of glacial acetic acid is used) followed by stirring the

\section{Refining of Used Engine Oil}

mixture in a closed container at room temperatures for a period of 1 hour. The mixture is then left aside for a few hours at room temperature(12-14Hours).[1]

2)Centrifugation:The mixture then undergoes centrifugation (3000 RPM) for a period of 1 hour which involves the application of centrifugal force to separate the base oil from the contaminants.[1]

3) Mixing with kaolinite:The separated base oil is mixed with kaolin- ite with appropriate ratios(For 2 litres of used engine oil $80 \mathrm{gm}$ of Kaolinite is used) for a period of 1 hour.and is then allowed to settle for a duration of 30 minutes. Kaolinite is added to remove the dark color and smell, which result from oxidation of some components of the oil. Followed by dehydration.[1]

4) Dehydration:The used Lubricating oil usually contains aromatics and water content etc,heating at high temperature to remove emulsified water, mixed lighter fractionators fuel oils in the heating container. In the dehydration, heating takes place at $110 \Rightarrow \mathrm{C}$ (approx.) where the entire water content is removed. [2]

5)Centrifugation:The mixture then undergoes centrifugation (3000 RPM) for a period of 30 minutes. Thus a black dark sludge at the bottom of the container is obtained. Followed by bleaching using activated carbon which is done to adjust the $\mathrm{PH}$ of of the oil to neutrality.[1]

6) Bleaching using activated Carbon:At this stage the oil is heated to a temperature of $120 \Rightarrow \mathrm{C}$ and then transferred to the mixing chamber where activated charcoal is introduced and it is mixed for about 10 minutes

and is then allowed to settle for a period of 10 minutes followed by a centrifuging(3000 RPM) for a period of 1 hour wherein the residue settles at the bottom which is then discarded and a dark red transparent base oil is produced.which is then stored in the base oil container.[3]

\section{DESIGN AND FABRICATION}

The design and fabrication of oil refining unit was made as an effort in find- ing environment-friendly means of waste oil refining .It is an alternative solution to the huge imports of engine oil.It is a prototype/laboratory scale model that will serve as the baseline in developing commercial oil refining plants. The main components are the frame,used oil storage conatainer,glacial acetic acid container,mixing chamber,kaolinite/Bleaching chamber,heating chamber and base oil storage container.

\subsection{Factors to be considered for a Reliable Design}

To ensure the safety is not compromised and the design is economic at the same time following factors are to be kept in mind:

1. Strength should be greater than stress induced

2. Simplicity of Design

3. Safety \& Convenience of control

4. Low Cost

\section{Good Appearance}

\subsection{Conceptual Design of oil Refining Unit}

The following shows the photos of the CAD Model of the oil refining unit designed on the solidworks software 2014 and of the working model.The design was made taking into consideration of cost,simplicity and avoiding complexity of fabrication. 


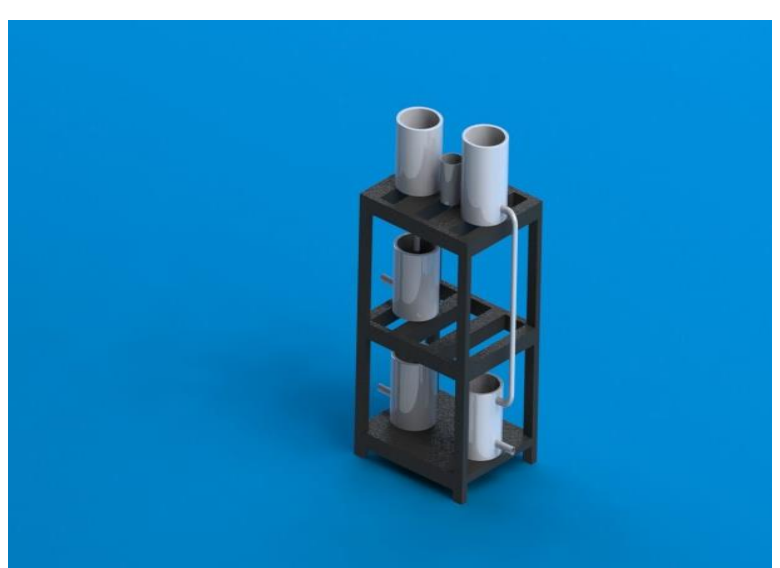

Figure 4.1: Conceptual Design of Oil Refining Unit

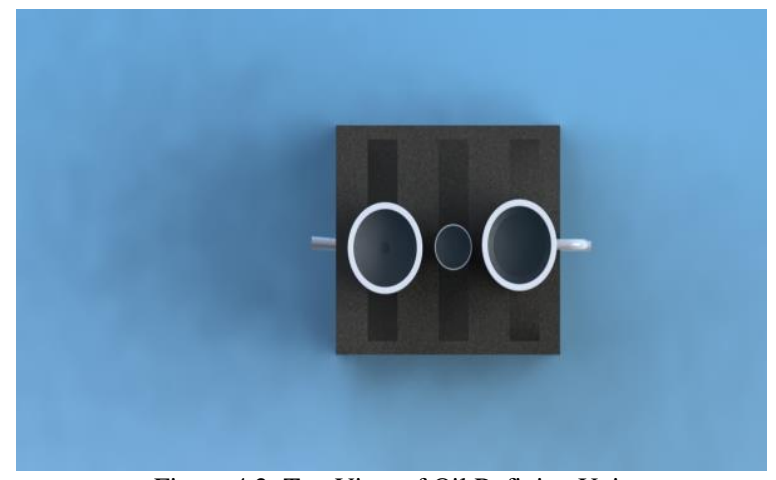

Figure 4.2: Top View of Oil Refining Unit

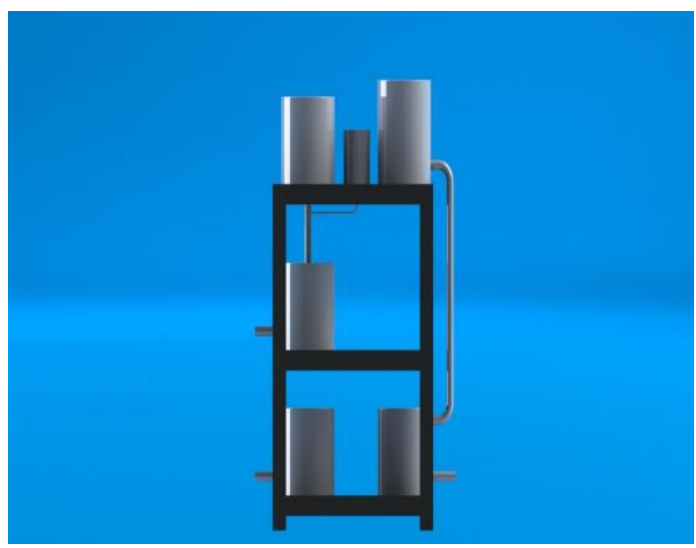

Figure 4.3: Front View of Oil refining Unit

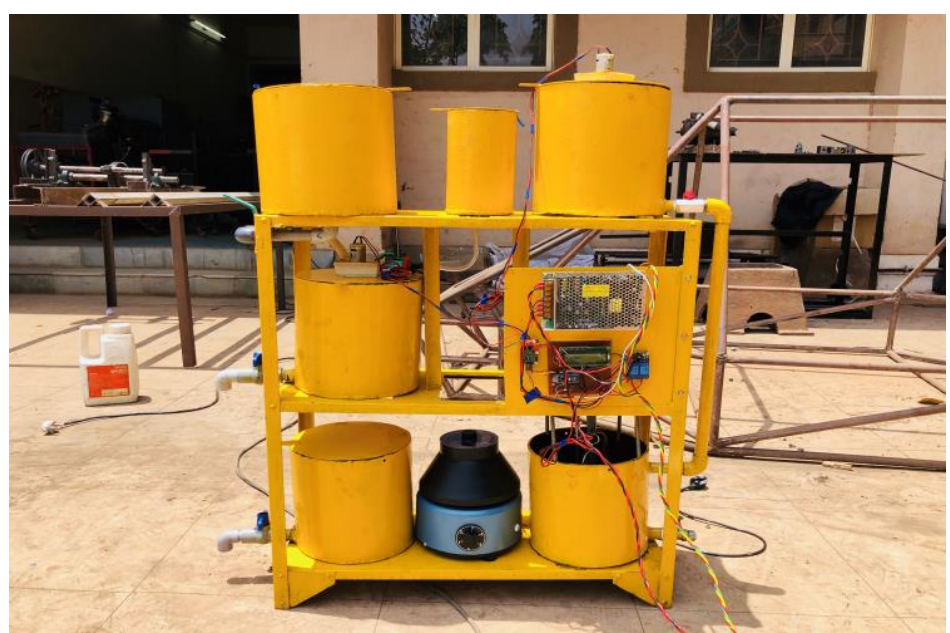

Figure 4.4: Working Model of Oil Refining Unit(5Litres Capacity)

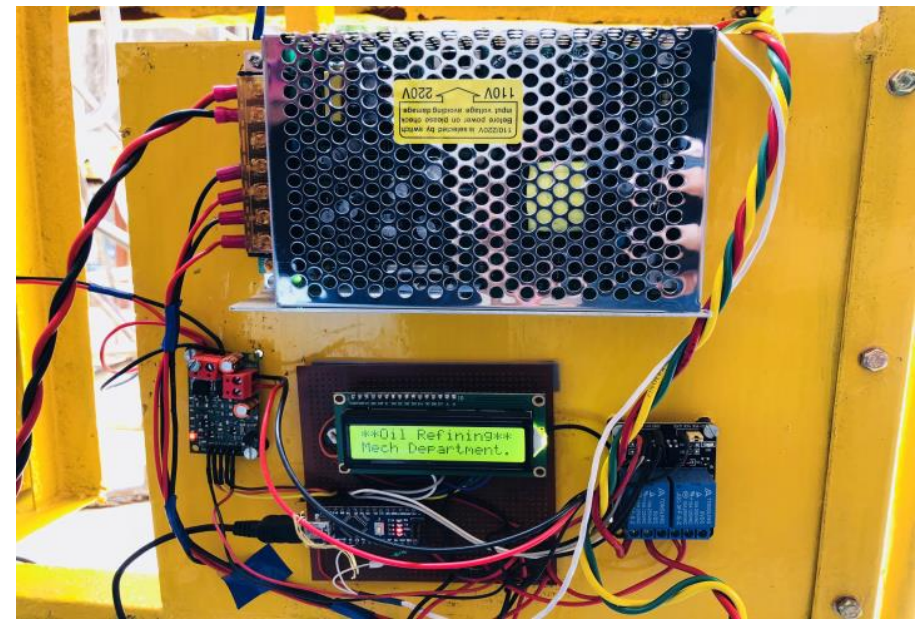

Figure 4.5: Electronic Assembly(Working Model)

\subsection{Conceptual Design of Centrifuge}

The following shows the photos of the CAD Model of the Centrifuge de- signed on the solidworks software 2014 and of the working model.The design was made taking into consideration of cost,simplicity and avoiding complexity of fabrication.

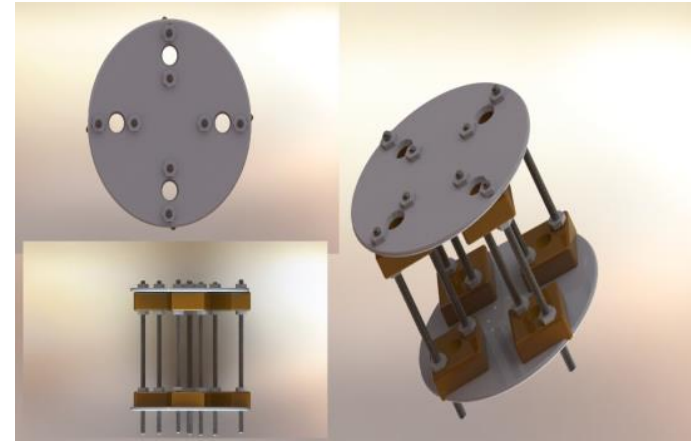

Figure 4.6: Conceptual Design of Centrifuge

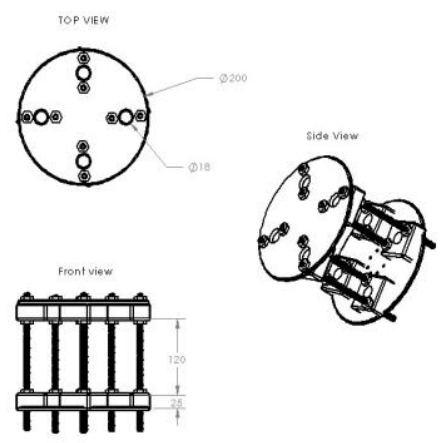

Figure 4.7: Dimensions of the Centrifuge

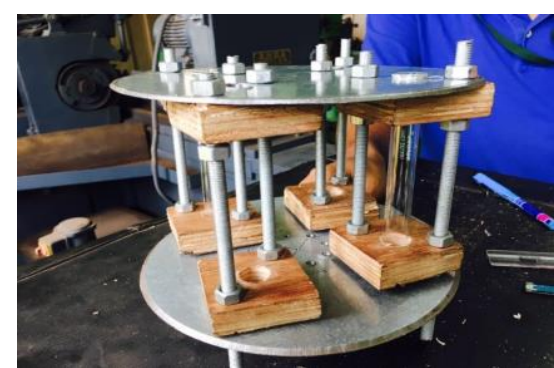

Figure 4.8: Centrifuge(Working Model) 


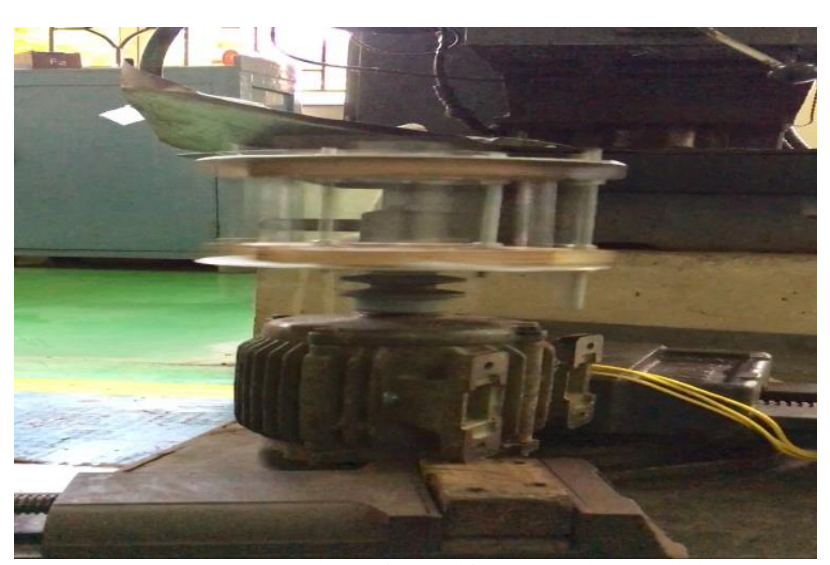

Figure 4.9: Assembly of Centrifuge(Working Model)

\subsection{Conceptual Design of Frame}

The following shows the dimensions of the frame,the frame takes the entire load of the oil refining unit hence the factor that the strength should be greater than the stress is taken into consideration.

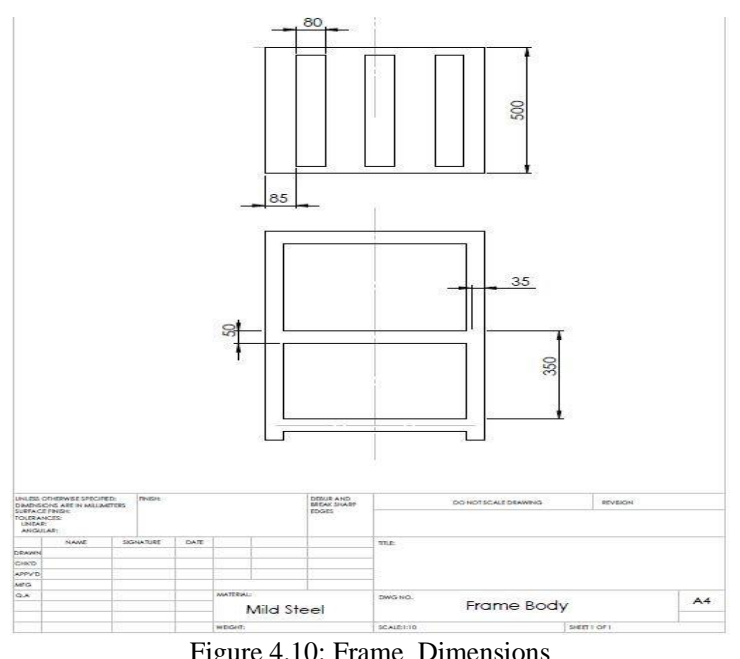

\subsection{Conceptual Design of Containers}

The containers were designed on the solidworks software 2014, the follow- ing shows the dimensions of the containers, these containers are used as the used oil storage container,kaolinite/Bleaching mixing chamber,mixing chamber,heating chamber and base oil storage container.The capacity of the the containers is 5 litres. There are total 5 containers of the same di- mensions and capacity as shown below.

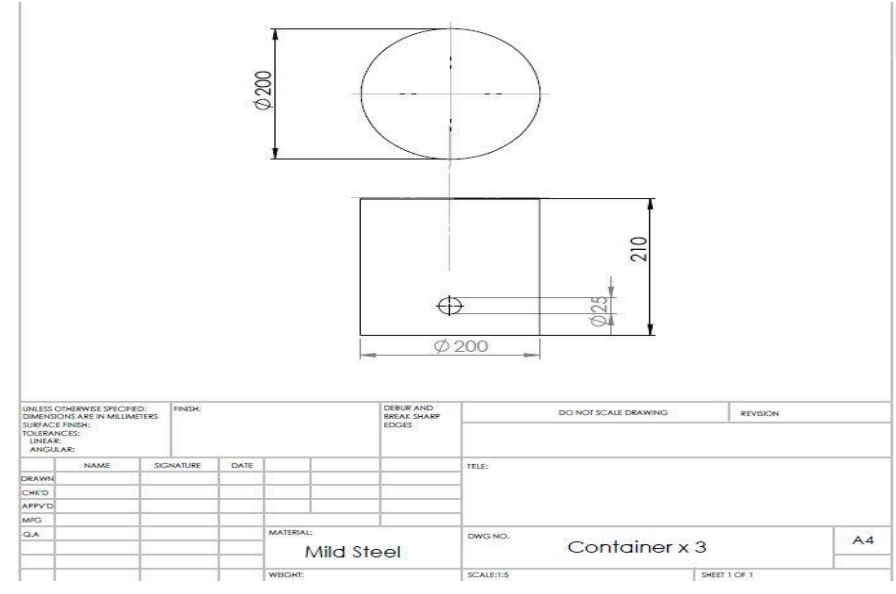

Figure 4.11: Dimensions of Containers

\subsection{Conceptual Design of Glacial Acetic Acid Container}

The Glacial Acetic Acid container was designed on the solidworks soft- ware 2014,the following shows the dimensions of the glacial acetic acid container,the capacity of the the containers is 1 litre.There is only 1 con- tainer of the following dimensions.
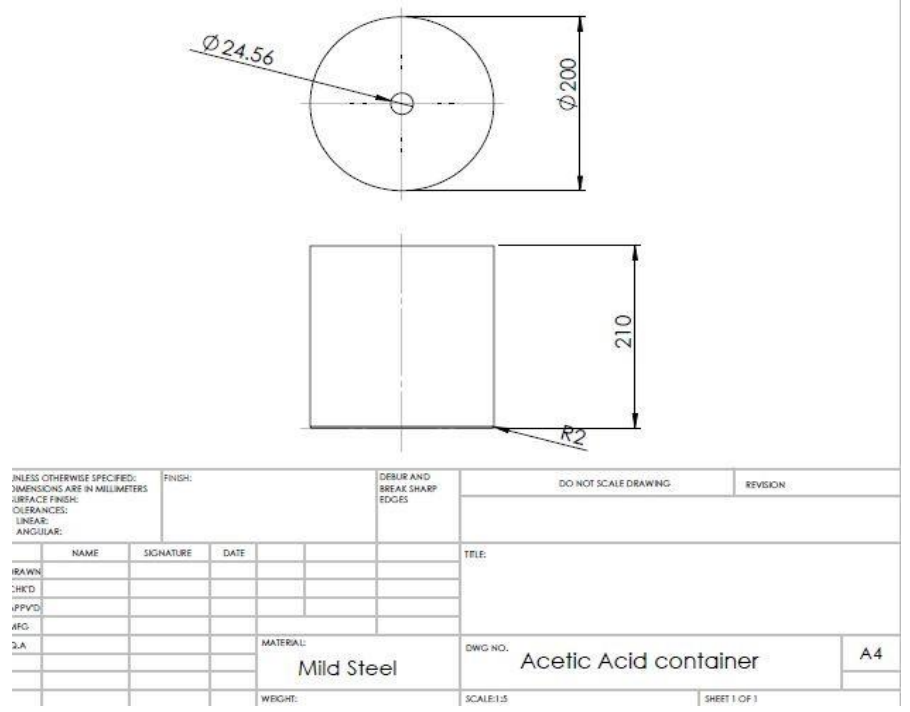

Figure 4.12: Dimensions of Glacial acetic acid Container

\section{COMPONENTS OF THE PROJECT}

The main components designed and fabricated for the refining process are as follows

1.Base Frame.

2.Mixing container,

3.Kaolinite/Bleaching Container,

4.Heating container

5.Auxiliary Components

\subsection{Base Frame}

Frame is the base of the machine. The frame is fabricated and is strong enough to sustain the load of all the containers,piping,valves.Apart from sustaining the weight the frame should be light and cost minimum consid- ering all the factors mild steel was chosen. The formability or 
workability of the mild steel is very good and the design of the frame has been done in SOLID WORKS 2014.

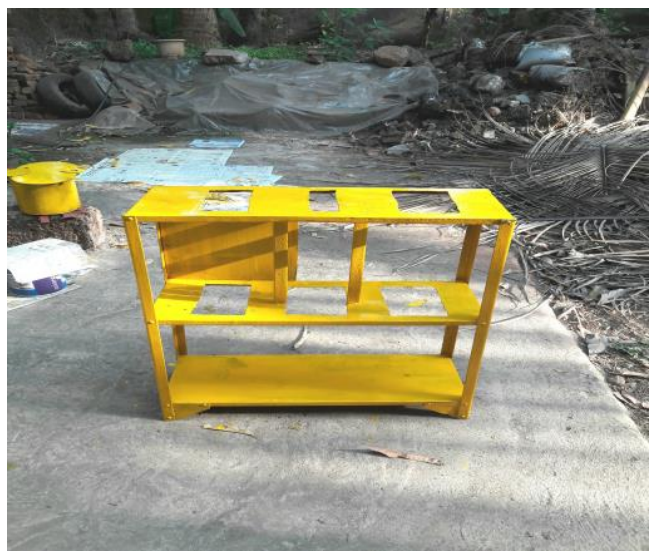

FIGURE 5.1: BASE FRAME

\subsection{Mixing Container}

This is the container used to mix the used engine oil and the acetic acid in appropriate proportions( For 2 litres of used engine oil $160 \mathrm{ml}$ of glacial acetic acid is used ) where mixing process will be initiated for a span of 1 hour.

\subsection{Kaolinite/Bleaching Container}

Here the oil is being agitated with kaolinite for a span of 1 hour and is then allowed to settle for a duration of 30 minutes ./The oil is mixed with activated charcoal for a period of 10 minutes followed by a settling time of 10 minutes.

\subsection{Heating Container}

This container has an industrial oil immersion heater which heats the oil uniformly and efficiently thus making it free from its long chain burnt car- bon atoms, and also helps in removing the humidity and moisture content present in the used engine oil.

\subsection{Auxiliary Components}

The Auxiliary components used in the oil refining unit are Arduino Board,Serial LCD,Motor Driver,Relays,SMPS,DC Motor,Centrifuge, Oil Immersion Heater and Resistance Temperature detector(RTD).

\subsubsection{Arduino Board}

Arduino is an open source, computer hardware and software company, project, and user community that designs and manufactures single-board microcontrollers and microcontrollers kits for building digital devices and interactive objects that can sense and control objects in the physical world. Arduino board designs use a variety of microprocessors and controllers.

\subsubsection{Serial LCD}

The interface contains only 3 pins, $+5 \mathrm{~V}, \mathrm{GND}$ and $\mathrm{RX}$. It uses only one microcontroller pin, and so it saves lots of hardware complexities and time. Upto 8 custom characters can be defined and used easily.

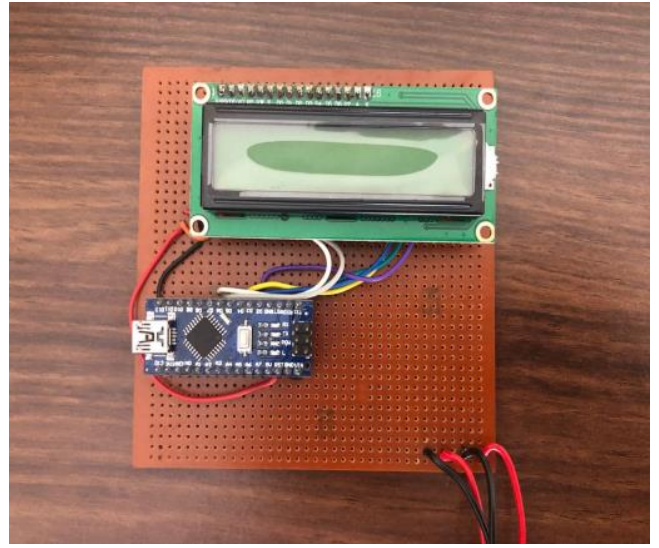

Figure 5.2: Serial LCD

\subsubsection{Motor Driver}

It comes up with a simple TTL/CMOS based interference that can connect directly to the IOs of an MCU.It has a breaking feature that can guarantee immediate halt on the shaft of the motors in most high power applications and also includes protection circuitry to avoid any electrical fluctuations affecting the normal operations of an MCU.

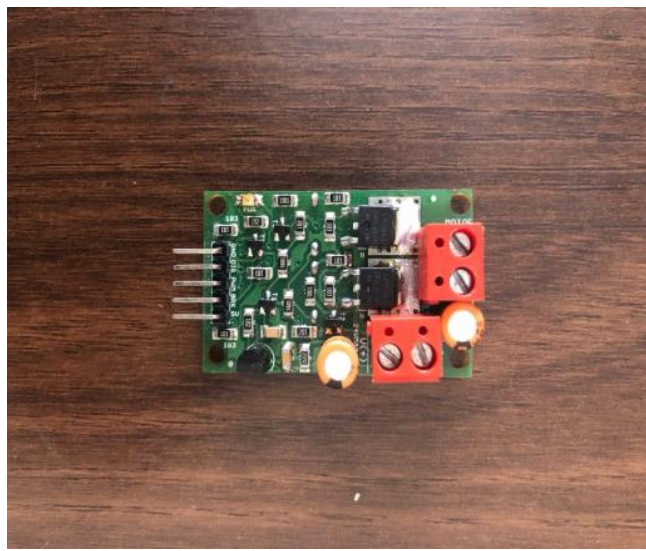

FIGURE 5.3: MOTOR DRIVER

\subsubsection{Relays}

Relays are switches that open or close electromechanically or electroni- cally.It's a 5V relay a normally open and one normally closed contact,the control pins with pull-down circuit,prevent floating relay malfunction.

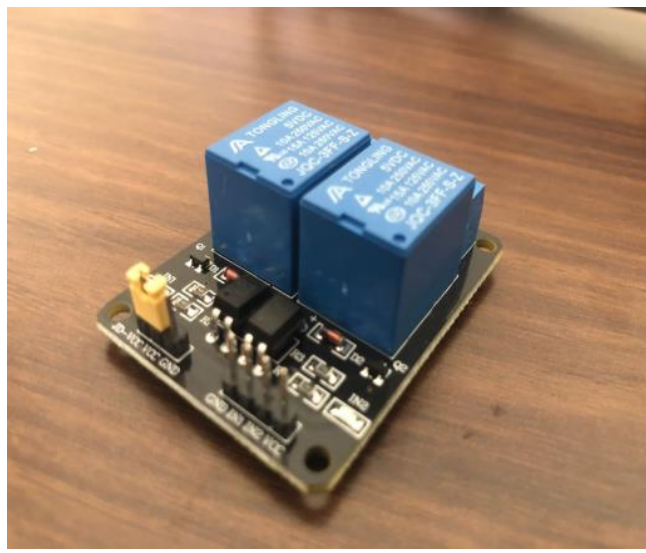

Figure 5.4: Relays 


\subsubsection{SMPS}

SMPS stands for switch-mode-power-supply.It rectifies the $\mathrm{AC}$ input per-forms the power factor correction and then converts the output into one or more lower voltage DC outputs.

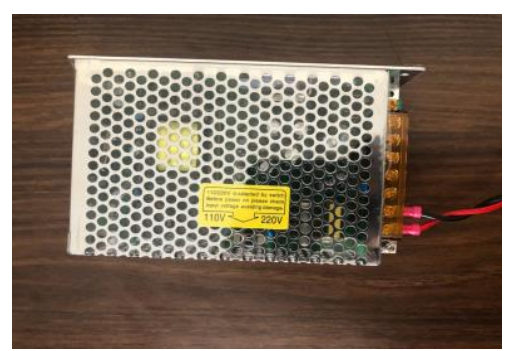

\subsubsection{MOTOR}

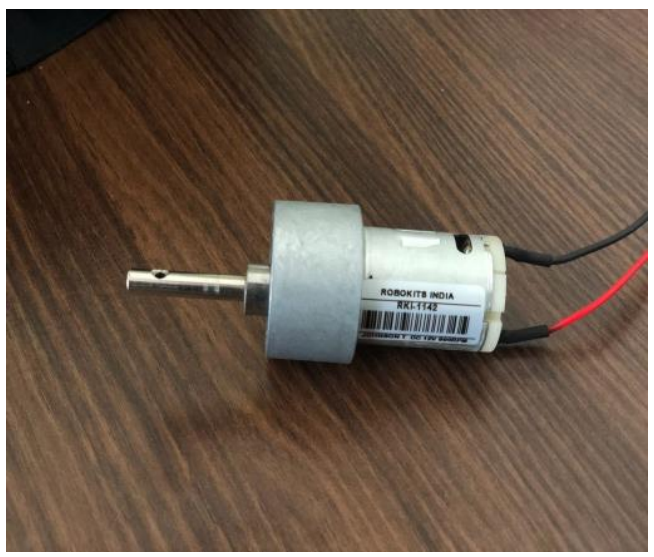

Figure 5.6: DC motor

IT IS A $12 \mathrm{~V}$ DC MOTOR WITH A METAL GEARBOX WITH 300 RPM

\subsubsection{CENTRIFUGE}

THE EQUIPMENT IS DRIVEN BY A MOTOR,WHICH SPINS LIQUID SAMPLES AT HIGH SPEED. IT WORKS ON THE PRINCIPLE OF SEDIMENTATION WHERE THE CENTRIPETAL ACCELERATION IS USED TO SEPARATE SUBSTANCES OF GREATER AND LESSER DENSITY.

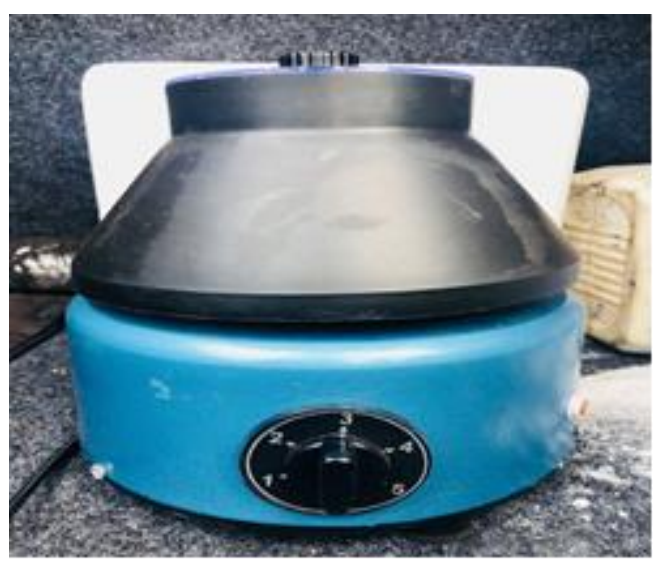

Figure 5.7: Centrifuge

\subsubsection{Oil Immersion Heater}

This works on the principle of joule heating.It has an electrical resistance heating element encased in a tube which converts the electrical energy into heat energy,a temperature sensor triggers a thermostat to control the temperature of the oil.

\subsubsection{Resistance Temperature Detector(RTD)}

RTD Pt100 is a device with a typical resistance of 100 ohm at $0 \Rightarrow$ (it is called Pt100). It changes resistance with a change in temperature. They are used in a variety of applications, including temperature measurement. They have been used for many years to measure temperature in laboratory and industrial processes,and have developed a reputation for accuracy, repeatability.

\section{FABRICATION PROCESS}

The Fabrication processes were carried out at Don Bosco College of Engi- neering Workshop.The various fabrication and machining operations car- ried out are cutting the pipes to required dimensions, welding the pipes to the container,drilling holes for the container,cutting of sheets,threading of pipes and grinding and finishing.

\subsection{Fabrication of oil Refining unit parts}

The following Parts were fabricated at the Workshop
1. Frame
2. Containers
3. Piping
4. Centrifuge

\subsection{Fabrication and Machining Operations}

\subsubsection{Cutting the Pipes to Required Dimensions}

PVC pipe of $\rightarrow$ inch diameter was cut using hacksaw blade for different lengths of 3 inches (4 Nos.), 4 inches (4 Nos.) and MS pipe of $\rightarrow$ inch diameter was cut for different lengths of 55inches and 4 inches ( 2 Nos.) and 2 inches ( 2 Nos.) and level pipe of 12 inches was cut to connect the glacial acetic acid container and mixing container.

\subsubsection{Welding Pipes to Container}

Electric Arc Welding was used:

To weld the MS pipes to the kaolinite/Bleaching container and the heating container.

To weld the cover plates at the bottom of the container to make it leak proof.

To weld the $10 \times 12$ inches sheet metal panel to the Base frame.

\subsubsection{Drilling Holes for Container}

Holes of different diameters were drilled on the containers using the Verti- cal Drilling Machine,the containers were placed on the regular work table and clamped to it using a G- 
clamp. Holes of different diameters were then drilled to it using drill bits of diameter $28 \mathrm{~mm}, 24 \mathrm{~mm}, 8 \mathrm{~mm}, 5 \mathrm{~mm}$ and $3 \mathrm{~mm}$.

\subsubsection{CUTTING OF SHEETS}

MS SHEET METAL WAS CUT IN CIRCULAR SHAPES OF DIAMETER 200MM AND 10MM FOR THE BOTTOM AND TOP OF THE CONTAINERS FROM A MS SHEET METAL USING A GRINDER, ALSO A 10X12INCHES RECTANGULAR SHAPED SHEET METAL WAS CUT FOR THE ELECTRONIC PANEL.

\subsubsection{THREADING OF PIPES}

THREADS WERE GENERATED ON A LATHE MACHINE FOR ITS ACCURACY AND FOR ITS VERSATILITY. THREADS WERE GENERATED ON A $\leadsto$ INCH MS PIPE HAVING THREAD PITCH LENGTH OF 0.07143 AND 14 THREADS PER INCH.

\subsubsection{Grinding and Finishing}

The stress concentration causes cracking which can be eliminated by using the grinding technique, the grinding machine has a grinding wheel disc which is composed of an abrasive compound which can easily remove the hard and brittle materials which are difficult to remove by cutting tools and also provides the required surface finish.

\section{PROPERTIES AND TESTING OF OIL}

The Testing of the following properties of used engine oil and refined engine oil was sucessfully carried out at FAN SERVICES(Materials \& Product Testing Laboratory)Satpur,Nashik.

\subsection{Kinematic Viscosity}

Kinematic Viscosity: Viscosity is a measure of the resistance to flow and may be regarded as the internal or fluid friction of the lubricating oil. It is a single most important property of a lubricant and must be known when formulating lubricants. It is a key measurement for detecting a number of used oil conditions including fuel dilution, oxidation and contamination. Oxidation products and contaminants such as soot, dirt, glycol and water cause viscosity increase while fuel dilution and shearing of viscosity index improver in multi-grade oils cause viscosity decrease. Kine- matic viscosities of the used and re-refined oil samples were measured at

$40 \Rightarrow \mathrm{C}$ and $100 \rightarrow \mathrm{C}$ with Canon-Fenske Routine viscometers or Canon-Fenske Opaque viscometers according to ASTM D 445 standard test methods for transparent and opaque liquids respectively.

Test Procedure (ASTM D 445) Kinematic viscosity is determined by measuring the time for a volume of oil to flow under gravity through a calibrated glass capillary viscometer. The dynamic viscosity can be obtained by multiplying the measured kinematic viscosity by the density of the oil.

\subsection{Viscosity Index}

Viscosity Index: Viscosity index (VI) is the viscositytemperature re- lationship and is an arbitrary number indicating the degree of change of viscosity of oil with change in temperature. It is one of the most criti-cal factors to consider for selecting lubricating oil for use in an internal combustion engine since the oil must function over a wide range of tem- perature. For oils of similar kinematic viscosity, the higher the viscosity index the smaller is the effect of temperature on its kinematic viscosity.

Test Procedure (ASTM D 2270) Viscosity index was determined from kinematic viscosities at $40 \rightarrow \mathrm{C}$ and $100 \rightarrow C$. The calculation of viscos- ity index from kinematic viscosities at $40 \rightarrow \mathrm{C}$ and $100 \rightarrow \mathrm{C}$ is exact, and no precision limits can be assigned to this calculation. The accuracy of the calculated viscosity index is dependent only on the accuracy of the origi- nal viscosity determination. Test Method D 445 has a stated repeatability limit of $0.35 \%$ and a reproducibility limit of $0.70 \%$.

\subsection{Moisture Content}

Moisture Content: Moisture is generally referred to as a chemical con- tamination when suspended in engine oils. water created in engine oil is a result of: absorbing moisture directly from the air (oil is hygroscopic), condensation (humid air entering oil compartments), heat exchanger (corroded or leaky heat exchangers), combustion (fuel combustion forms water which may enter the lubricant oil through worn rings), oxidation (chemi- cal reaction) and neutralization (when alkalinity improvers neutralize acids formed during combustion), and free water entry (during oil changes)

Test Procedure (ASTM D 5349) The Oil sample is weighed and air dried to equilibrate it with the atmosphere. The residual moisture is then determined using the Drying Oven Method. Air drying and Residual moisture are combined to report gross moisture.

\subsection{Density}

Density: Density is the ratio of the mass of volume of Oil to the mass of the same volume of water and depends on two temperatures, at which the mass of the oil and the water are measured. Density is influenced by the chemical composition of the oil. An increase in the amount of aromatic compounds in the oil results in an increase in the density, while an increase in the saturated compounds results in a decrease in the density. Density is an important quality indicator for automotive oils where it affects pricing storage, handling and combustion.

Test Procedure (ASTM D 1298) The sample is brought to the pre- scribed temperature (standard reference temperature of $15 \rightarrow C$ ) and trans- ferred to a cylinder at approximately the same temperature. The appro- priate hydrometer is lowered into the sample and allowed to settle. After temperature equilibrium has been reached, the hydrometer scale is read, and the temperature of the sample is noted. If necessary the cylinder and its contents may be placed in a constant temperature bath to avoid excessive temperature variation during the test. 


\subsection{Flash Point}

Flash Point: The flash point of an engine oil is the lowest temperature to which the oil must be heated under specified conditions to give off sufficient vapor to form a mixture with air that can be ignited spontaneously by a specified flame. The flash point of engine oil is an indication of the oil's contamination. A substantially low flash point of an engine oil is a reliable indicator that the oil has become contaminated with volatile products such as gasoline

Test Procedure (ASTM D 92) The test cup is filled to a specified level with oil sample. The temperature is rapidly increased at first, and then at a slow constant rate as the flash point is approached. At specified intervals a small test flame is passed across the cup. The lowest temper- ature at which the vapors above the surface of the liquid ignites is taken as the flash point.

\subsection{Color}

Color: New oil is amber in color as engine oil gets darker it can indicates high heat, contaminants, the presence of additives that cause the oil to darken during normal use.

Test Procedure (ASTM D 1500) Using a standard light source, a oil sample is placed in the test container and compared with colored glass disks ranging in value from 0.5 to 8.0. When an exact match is not found and the sample color falls between two standard colors, the higher of the two colors is reported.

\section{EXPERIMENTS CONDUCTED IN LAB}

A total of 3 experiments were conducted out of which experiment 3 showed comparatively better results.

\subsection{Experiment 1}

Oil was taken in a bottle and mixed thoroughly for 15 minutes. $5 \mathrm{ml}$ of oil was added in a test tube and mixed with glacial acetic acid this was stirred for $20 \mathrm{mins}$.

Conclusion: The black engine oil turned into a fainted brown colour, there was also a change in colour of glacial acetic acid from colourless to light red colour.

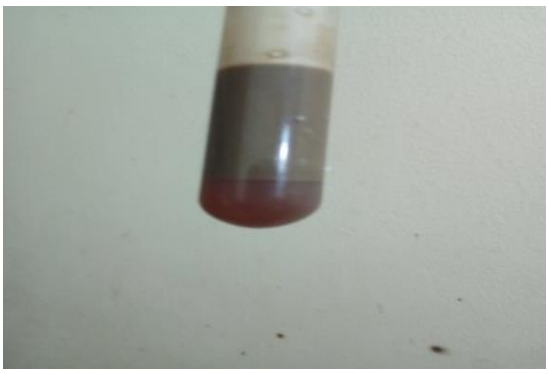

Figure 8.1: Experiment No.1

\subsection{EXPERIMENT 2}

10 ML OF WASTE ENGINE OIL WAS TAKEN AND MIXED WITH 1.6ML ACETIC ACID.LATER 0.4GMS OF KAOLIN CLAY WAS ADDED AND MIXED THOROUGHLY FOR 30 MINUTES AND HEATED TO A TEMPERATURE OF $80 \rightarrow \mathrm{C}$.

CONCLUSION: 2 LAYERS WERE OBSERVED, THE BASE OIL (YELLOW) BEING AT THE TOP LAYER.

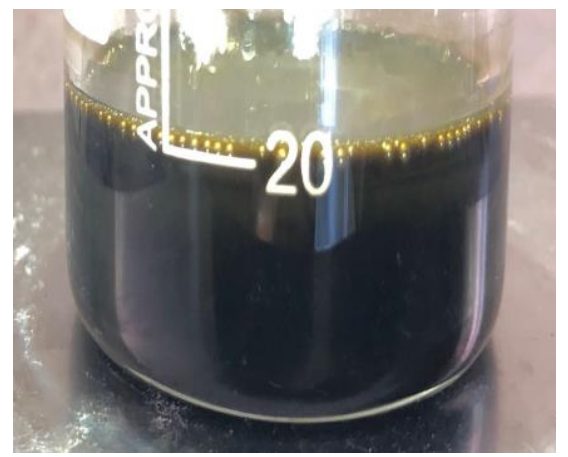

FIGURE 8.2: EXPERIMENT NO.2

\subsection{Experiment 3}

$10 \mathrm{ml}$ of waste engine oil was taken and mixed with $1.2 \mathrm{ml}$ acetic acid for 60 minutes and kept for 24 hours. After 24 hours, centrifugation was performed for 1 hour.

Conclusion: The dark black oil turned out to be light black also after centrifugation the contaminants got settled at the bottom of the test-tube.

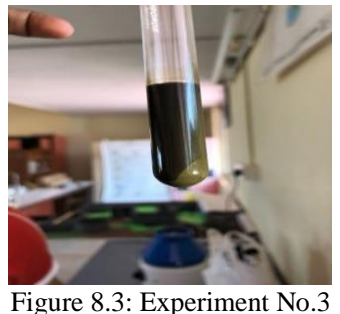

\section{LAB TESTING REPORTS}

The Testing of the following properties of used engine oil andrefined engine oil was sucessfully carried out at FAN SERVICES(Materials \& Product Testing Laboratory)Satpur,Nashik. The testing of the following properties was carried out sucessfully, kinematic viscosity at $40 \Leftrightarrow$ C,kinematic viscos- ity at $100 \Leftrightarrow$ C,density at $25 \mapsto \mathrm{C}$, density at $15 \rightarrow \mathrm{C}$,specific gravity,moisture content,flash point,colour,colour rating and viscosity index.The lab testing reports of used engine oil and refined engine oil were then compared with the standard base oil(ASTM).The refined engine oil showed comparable results as shown by the traditional methods.Hence based on the lab test- ing reports this process can be implemented in the pre-treatment section of the waste engine oil refining for commercial use. 


\subsection{Report of Waste Engine oil}

The Testing of the following properties of used engine oil was carried out in order to determine the properties and contamination levels of used engine oil which was later compared with the refined engine oil.

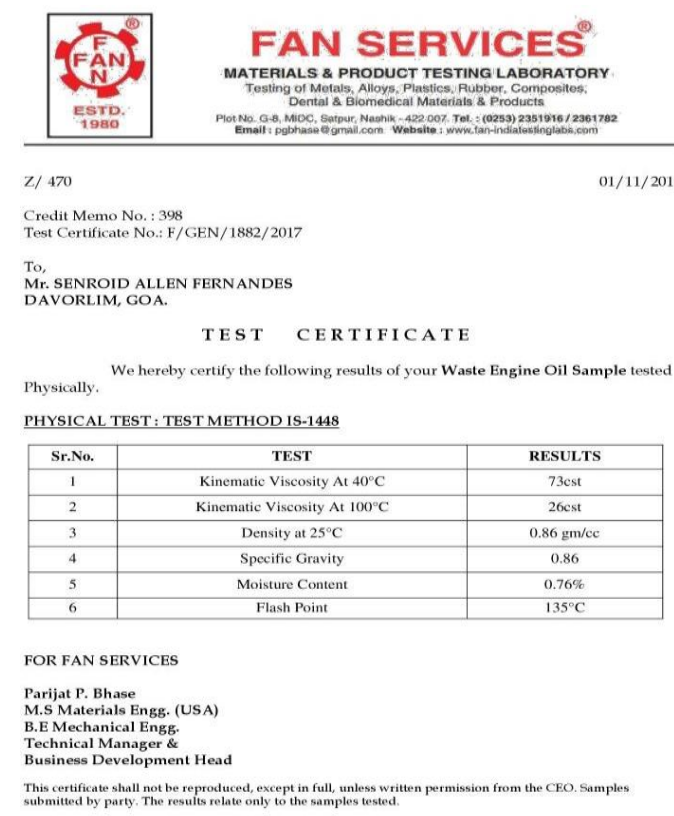

FIGURE 9.1: TEST REPORT OF WASTE ENGINE OIL

\subsection{Report of Refined Engine oil}

The Testing of the following properties of refined engine oil was carried out in order to determine the properties and reduction in contamination levels of refined engine oil and was compared with the used engine oil.

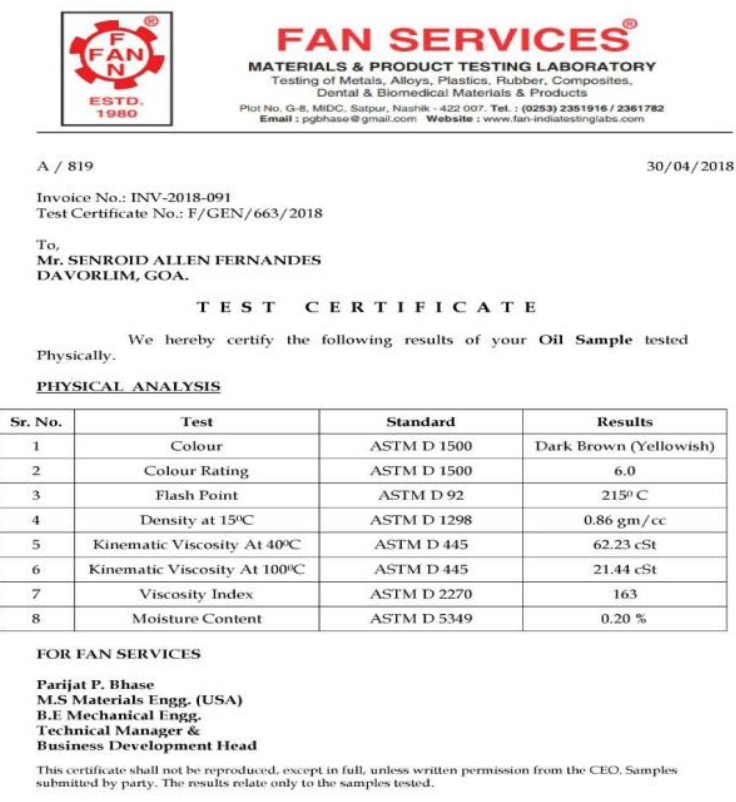

Figure 9.2: Test Report of Refined Engine oil

\subsection{Comparison Table}

The Sample of waste Engine oil and Refined oil was tested as per ASTM Standards for colour,Flash Point,Density,kinematic viscosity at $40 \rightarrow \mathrm{C}$ and

$100 \Rightarrow$ C,viscosity Index and Moisture Content and the results were compared to the standard Base oil Group 1(ASTM Standards).Testing of the used engine oil and the refined engine oil was carried out sucessfully,the refining of used engine oil with acetic acid-clay treatment was carried out sucess- fully resulting in red transparent colour base oil.This process of refining of used engine oil did not emit poisonous gases like sulphur dioxide to the atmosphere as compared to the traditional refining methods.In addition the glacial acetic acid has less impact on the processing equipment com- pared with sulphuric acid used by the traditional refining methods.This method produces base oil comparable to that produced using conventional methods.Based on the comparison table it can be stated that the refining of used engine oil was sucessfully carried out and this process can be im- plemented in the pre-treatment section of the oil refining for commercial use.

\section{X.CONCLUSION}

Testing of properties of used Engine oil was successfully carried out.

Testing of properties of Refined Engine oil was successfully carried out.

The refining process was carried out and transparent red color Base oil was obtained.

Refining of used engine oil with acid-clay treatment was carried out successfully resulting in Red transparent colour base oil.

This Project has shown that used engine oil can be recycled by using glacial acetic acid. This method produces base oil comparable to that produced using conventional methods.

Optimum conditions for recycling used engine oil using this method are room temperature. The process for recycling is simple, as it only requires mixing at room temperature, settling, centrifugation and fi- nally mixing with kaolinite and mixing with activated charcoal.

This new process of refining of used engine oil did not emit poisonous gases like sulphur dioxide to the atmosphere. In addition, glacial acetic acid has less negative impact on the processing equipment as compared with sulphuric acid.

\section{Outcome}

The device is Cost efficient.

This device is cost efficient as it does not use high end cost technol- ogy such as stripping kettle,thin flim evaporator,high vaccum molecular distilation and vaccum skid.

It is Environment-Friendly.

This new process of refining of used engine oil did not emit poisonous gases like sulphur dioxide to the atmosphere. In addition, glacial acetic acid has less negative impact on 
the processing equipment as compared with sulphuric acid.

It is Portable to shift.

This device is very light in weight and hence it can be easily moved to different places.

The above process shows better quality of regenerated oil.

This process of refining of used engine oil was performed and the refined engine oil was tested for various properties in the lab and it was found that it showed comparable results as shown by the traditional methods of refining of used engine oil.Hence this process of refining used engine oil can be implemented in the pre-treatment section of the oil refining plants for commercial use.

\section{Future Scope}

In future this project can be improved by the following ways:

Addition of vaccum distillation wherein the oil is passed through a vaccum distillation column, under higher vacuum and temperature, to distill off lighter oil. The undistilled oil is subjected to high vac- uum, high temperature in an induction heated plant. The distilled oil will have a viscosity of about SAE 40 at ambient.

Addition of oil pumps and a 10 litre capacity centrifuge including solenoid valves would automate the entire process.

\section{REFERENCES}

[1] Recycling of Waste Engine Oils Using a New Washing Agent by Ihsan Hamawand, Talal Yusaf, and Sardasht Rafat.

[2] Merai Yash P." Re-refining of used lubricating oil" International Jour- nal of Scientific \& Engineering Research, Volume 6, Issue 3 , March-2015

[3] Isah, A. G., Abdulkadir, M., Onifade, K. R., Musa, U., Garba, M. U., Bawa, A. A and Sani, Y." Regeneration of Used Engine Oil" Proceed- ings of the World Congress on Engineering 2013 Vol I, WCE 2013, July

[4] Waste and used Lube oil Refining Process almost zero clay SLMAC (SHORT PATH DISTILLATION) by Arslan Enginery.

[5] Guide to ASTM Test Methods for the Analysis of Petroleum Products and Lubricants 2nd Edition by R. A. Kishore Nadkarni.

[6] Ramon, Manoel Carlos. "Arduino IDE and Wiring Language." Arduino

[7] Pair, C. "C++ Programming, Programming Languages and Program- ming Methods.” Psychology of Programming (1990): 9 19. Web.W.-K. Chen, Linear Networks and Systems. Belmont, CA: Wadsworth, pp. 123-135, 1993. 